\title{
An Analysis of Code Switching and Code Mixing in Fashion Program "I Look" on Net Tv
}

\author{
Renita Wati \\ Pendidikan Bahasa Inggris STKIP Nurul Huda Sukaraja \\ Renitawati30@gmail.com
}

\begin{abstract}
This study focuses on the form and function of code-switching and code-mixing used in the Fashion Program "I LOOK " on NET TV. This study is aimed to answer the following problem: 1 . What is the forms of code-switching and code-mixing used in the Fashion Program "I LOOK" on NET TV, and What are the functions of code-switching and code-mixing in Fashion Program "ILOOK" on NET TV. To answer the problem, the writer used descriptive qualitative research through theoretical triangulation the total data 72 unit of analys is of code-switching found that 22 data used tag switching, 18 data used inter-sentential switching, and 32 used intra-sentential switching. It showed that intrasentential switching is commonlyused by Kimmy Jayanti in the fashion program "I LOOK" on NET TV. Furthermore, 127 unit data analysis of code-mixing performed 74 insertions of words, 48 insertions of phrases, 2 insertions of hybrids, and 3 insertions of reduplications. Meanwhile, there is 250 function of code-switching and code-mixing used by Kimmy Jayanti in the fashin program "I LOOK" in NET TV. They are quotation 1, addressee specification 24, repetition 1 , interjection 31 , message qualification 12, personalization and objectivization 15 , and facility of expression 121.
\end{abstract}

Keywords: Code Switching, Code Mixing, Fashion Program.

\section{INTRODUCTION}

In daily interaction, people use language as their communication tool in the form of writing, spoke, and symbols. According to Wibowo (2001:3), language is a system of symbols that are meaningful and articulate sound (generated by the said tool) that are arbitrary and conventional, which is used as a means of communication by a group of human to give birth to feelings and thoughts. Humans are social beings who live in groups that have language, tradition, and culture. Therefore, it is impossible to communicate with others without language. People use language to gain an understanding of their willingness, needs, or orientation. In the way to communicate, many languages are used in the world. One of them is English. English is a global language (Crystal, 2003).

In general, People make a conversation not only use their language but also generally people often use more than one language in communication. Commonly, people develop some knowledge and ability in bilingual languages. According to Kridalaksana (2008:36), bilingualism is the use of two languages by an individual and community. This means that bilingualism is the two languages used by the speaker to interact with the speaker in different languages. Bilingualism is related to codeswitching and code-mixing because they are related to two different languages that are used in conversation.

Shite (2016:9) defines that code-switching as a means of communication involving a speaker alternating between one language and another at the level of the sentence. In another way, people may speak by mixing one language to another language to make the conversation running well. According to Wardhaugh (2006:106), code-mixing occurs when conversants use both languages to the other in the course of a single utterance. The speakers do not necessarily need to change the language but they mix the languages and use them together in a statement. This means that difference between code-switching and code-mixing is true that code-switching has a special, social pragmatic consequence while code-mixing does not. Both code-switching and code-mixing are usually used by Indonesian to attain good communication in a society. Code-switching and codemixing are not only used in direct interaction but also in program television. 
The television program from NET TV which features a Fashion program entitles "I LOOK". This program shows a lot of code-switching and code-mixing of languages, which gives a special attraction for the audience. The program was presented by Kimmy Jayanti. Kimmy Jayanti used both Indonesian as her mother tongue and English as the second language.

Based on the phenomena above, the researcher was interested in conducts research entitled " An Analysis of Code Switching and Code Mixing in Fashion Program 'I LOOK' in NET TV.

\section{Concept Code Switching}

People use language as a code to communicate with others. Code-switching is one aspect of the interdependence of language in a multilingual society. That is, in a multilingual society is almost impossible for a speaker in one language is pure without the slightest utilizing language or other language elements.

Many linguists make an explanation about code-switching. According to Suwito (1983: 68), code-switching is the event code transition from one code to another code. Besides that, according to Jendra (2012: 74), states that code-switching has become a common term for alternate use of two or more languages, or varieties of language, or even speech styles. It can be concluded that codeswitching is the alternate use of two or more languages within the same utterance. Code-switching is a change by a speaker or writer from one language or language variety to another. It may start one language and then change to another one in the middle of their speech, or even in the middle of the sentence.

\section{Concept Code Mixing}

Code mixing occurs in bilingual or generally in a multilingual situation. Code mixing occurs when a person uses a language speakers are dominant, supporting a speech interspersed with elements of other languages.

According to Kridalaksana in Purwanto (2002: 27), code-mixing is interference, and the use of language units from one language to another to expand the style of language or language variety: it includes the use of the word, clause, greeting, and so on. By the mean it, code-mixing occurs in a single sentence or utterance without breaking the grammatical rules of the mind language. Besides that Richards (2002: 80), code-mixing is a mixing of two code or language, usually without a change of topic. Based on all of the statement above, it can be seen that almost of those states have the same understanding of code-mixing that it is uses two or more language or mix from one code to another even within sometimes very short utterances without any change at all situation.

\section{Form of Code Switching and Code Mixing Form of Code Switching}

According to Jendra (2012: 75-77), there are three types of code-switching :

\section{a) Tag Switching}

Tag code-switching happens when a bilingual inserts a short-expression (tag) from a different language at the end of his/ her utterances.

b) Inter-sentential Switching Inter-sentential code-switching happens when there is a complete sentence in a foreign language uttered between two sentences in a base language.

c) Intra sentential Switching An intra- sentential code-switching is found when a word, a phrase, or a clause, of a foreign language, is found within the sentence in a base language.

\section{Form of Code Mixing}

Suwito (1985:78) classifies code-mixing into six kinds according to the linguistic elements that involved in code-mixing as follows:

a) The Insertion of Word

The insertion of words here means the language unit that stands on its own, it consists of free morpheme sand bound morphemes. 
b) The Insertion of Phrase

A phrase is a group of two or more related words that do not certain both as subject and a predicate.

c) The Insertion of Hybrid

Hybrid is a combination of word pieces, the combination pieces of Indonesian word, and English word.

d) The Insertion of Reduplication

Word reduplication is the repetition of some parts of the base (which may be the entire base) more than one word.

e) The Insertion of Idiom

Idioms are an expression that has a meaning different from that, of the individual meaning of each of its parts.

\section{Functions of Code-Switching and Code Mixing}

Code-switching and code-mixing have each function in communication. There are several functions according to Marasigan (1983:71) :

\section{a) Quotation}

According to Marasigan (1983:73), "subjects quoted themselves and others directly or indirectly to sound more credible to the addressees".

b) Addressee Specification

According to Marasigan (1983:73) stated that in this type of switch, it recognizes not only interacting members of the speech events but also recognizes that either language behaviors may be more than merely a matter of individual preference or facility, but also role relation.

c) Repetition

A message is repeated in the other code aimed to clarify what the speaker said, strengthen or emphasize the message, or mark the joke.

d) Interjection

According to Marasigan (1983:.81), an interjection is used to change the interaction from the "we" to "they" code or from the "they" to the "we" code. It means that the speaker used interjection to switch and mix the code. The interjection is words or expressions, which are inserted into a sentence to convey surprise, strong emotion, or to gain attention.

e) Message Qualification

According to Marasigan (1983:84) states that "Message qualification is to express the time of concept". She (1983) also explained that another large group of switches consist of qualifying constructions such as clauses, sentences, and phrases (verb and noun complements).

f) Personalization and Objectivization

Personalization and Objectivization are related to a statement of personal opinion, feeling, or knowledge; whether it refers to specific instances, or whether it has the status of generally known fact.

g) Facility of Expression

According to Marasigan (1983:90), explained that the text contains several instances "where the shift ... can only be interpreted as difficulty in finding the right word at the time of speaking or writing or merely as a sign of the subject's lack of familiarity with the style he is using".

\section{METHODOLOGY}

The research method is very important in conducting research, the researcher must apply an appropriate method. According to Moleong (2009:5,) "Qualitative research is a research which used a natural background to interpret the phenomena and it was done by involving various methods". Besides that, According to Moleong (2002:3), "qualitative methodology is a research procedure that produces descriptive data in written or oral words from people and observed act.

This research is descriptive qualitative research. The focus is on the description of form and function of code-switching and code-mixing in the fashion program "I LOOK" in NET TV that analyzes.

Collecting the data is very important to determine the result of the study. In this study, the procedure to be used in the collection is: (1) Watching and listening to " I LOOK " on NET TV to be selected. (2) Making transcription from the videos. (3) Encoding the collecting data. (4) Classifying the utterance either code-switching or code-mixing found in Fashion Program " I LOOK " in NET TV. (5) Analyzing the form and function of code-switching and code-mixing. 
In this study there are some steps to analyze the data : (1) Identification, (2) Coding, (3) Categorizing, (4) Classifying, (5) Producing an account.

\section{RESULT AND DISCUSSION}

\section{Tabel 1}

\section{Analysis of the use of code-switching and code-mixing in "Mix and Match Korean Style"} episode

\begin{tabular}{|c|c|c|c|c|}
\hline No & $\begin{array}{c}\text { The Data } \\
\end{array}$ & Lexical Form & Form & Function \\
\hline \multirow[t]{3}{*}{1.} & \multirow{3}{*}{$\begin{array}{l}\text { Wow keren keren banget style korea } \\
\text { zaman sekarang. Ih Kimcy jadi pengen } \\
\text { deh, tapi gimana ya, ok coba kimcy } \\
\text { browsing ah. }\end{array}$} & Style & G1 & $\mathrm{H} 7$ \\
\hline & & Ok & G1 & $\mathrm{H} 7$ \\
\hline & & Browsing & G1 & $\mathrm{H} 7$ \\
\hline \multirow[t]{4}{*}{2.} & \multirow{4}{*}{$\begin{array}{l}\text { Korean style sedang di gemari para } \\
\text { fashionista, baik pria maupun wanita, } \\
\text { buat kamu yang menyukai korean style } \\
\text { tapi masih bingung cara mix and } \\
\text { matchnya? Don't worry! Let's check this } \\
\text { out! }\end{array}$} & Korean style & G2 & $\mathrm{H} 2$ \\
\hline & & Matchnya & G3 & $\mathrm{H} 4$ \\
\hline & & Don't worry! & F1 & $\mathrm{H} 4$ \\
\hline & & $\begin{array}{c}\text { Let's check this } \\
\text { out! }\end{array}$ & F1 & $\mathrm{H} 4$ \\
\hline \multirow[t]{9}{*}{3.} & \multirow{9}{*}{$\begin{array}{l}\text { First Tuked it in. Jika kamu perhatikan } \\
\text { korean girl suka sekali dengan style } \\
\text { tuked it in yaitu dengan memilih skirt } \\
\text { ataupun hots pants dengan model hight } \\
\text { waist. Kamu bisa mix and match } \\
\text { tucked it in kamu dengan memilih } t \text {-shirt, } \\
\text { sweater, hingga kemeja, dan gaya ini } \\
\text { juga menjadi gaya favorit girl band } \\
\text { korea seperti Suzi dan Wo Soon }\end{array}$} & First Tuked it in & F2 & $\mathrm{H} 7$ \\
\hline & & Style tuked it in, & F3 & $\mathrm{H} 7$ \\
\hline & & Skirt & G1 & $\mathrm{H} 7$ \\
\hline & & Hots pants & G2 & $\mathrm{H} 7$ \\
\hline & & Hight waist & G2 & $\mathrm{H} 7$ \\
\hline & & $\begin{array}{l}\text { Mix and match } \\
\text { tucked it in }\end{array}$ & F3 & $\mathrm{H} 6$ \\
\hline & & T-shirt & G1 & $\mathrm{H} 7$ \\
\hline & & Sweater & G1 & $\mathrm{H} 7$ \\
\hline & & Girl band & G2 & $\mathrm{H} 2$ \\
\hline \multirow[t]{7}{*}{4.} & \multirow{7}{*}{$\begin{array}{l}\text { Next Oversized top. Oversized top } \\
\text { menjadi fashion item favorit di Korea, } \\
\text { dengan menggunakan atasan yang } \\
\text { kebesaran dan juga bawahan yang ketat, } \\
\text { kamu akan tampil gaya ala Korean } \\
\text { girl. Style korea yang satu ini akan } \\
\text { membut kamu menjadi sporty dan juga } \\
\text { cute. }\end{array}$} & $\begin{array}{l}\text { Next Oversized } \\
\text { top }\end{array}$ & F2 & $\mathrm{H} 7$ \\
\hline & & Oversized top & G2 & $\mathrm{H} 7$ \\
\hline & & Fashion item & G2 & $\mathrm{H} 7$ \\
\hline & & Korean girl & G2 & $\mathrm{H} 2$ \\
\hline & & Style & G1 & $\mathrm{H} 7$ \\
\hline & & Sporty & G1 & $\mathrm{H} 7$ \\
\hline & & Cute & G1 & $\mathrm{H} 7$ \\
\hline \multirow[t]{5}{*}{5.} & \multirow{5}{*}{$\begin{array}{l}\text { Tapi ada beberapa aksesoris yang bisa } \\
\text { kamu pilih untuk jadi pemanis penampilan } \\
\text { seperti round clear glasses, snapback, } \\
\text { atau hairband. Kamu akan kelihata simple } \\
\text { namun juga cute fashion people. }\end{array}$} & $\begin{array}{c}\text { Round clear } \\
\text { glasses }\end{array}$ & G1 & $\mathrm{H} 7$ \\
\hline & & Snapback & G1 & $\mathrm{H} 7$ \\
\hline & & Hairband. & G1 & $\mathrm{H} 7$ \\
\hline & & Simple & G1 & $\mathrm{H} 7$ \\
\hline & & $\begin{array}{c}\text { Cute fashion } \\
\text { people }\end{array}$ & F3 & $\mathrm{H} 2$ \\
\hline 6. & $\begin{array}{l}\text { Next drees with } t \text {-shirt. Jika kamu tidak } \\
\text { terlalu pede menggunakan drees with } t \text { - }\end{array}$ & $\begin{array}{l}\text { Next drees with t- } \\
\text { shirt }\end{array}$ & $\mathrm{F} 2$ & $\mathrm{H} 7$ \\
\hline
\end{tabular}




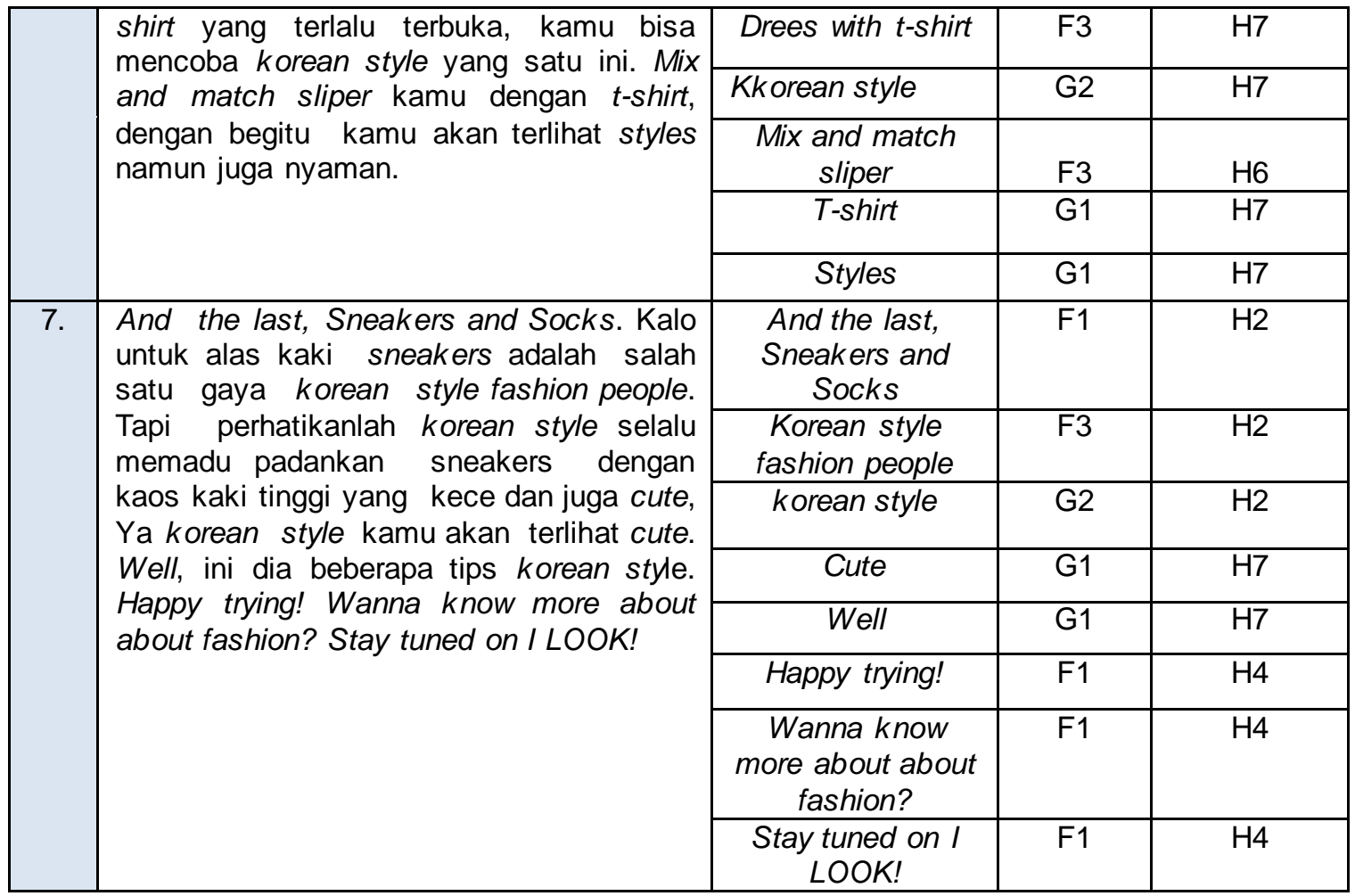

From the table above, it can be seen that there are 14 occurrences of code-switching, the type in which tag switching (F1) is 6, inter-sential (F2) is 3 and intra-sential (F3) is 5. Meanwhile there are 25 occurrences of code-mixing, the writer just found two types of code-mixing. They are the insertion of the word (G1) is 16, and the insertion of the phrase (G2) is 9. Then for the function of codeswitching and code-mixing the writer only found four functions in the "Mix and match Korean style" episode. They are addressee specification $(\mathrm{H} 2)$ is 6 , interjection $(\mathrm{H} 4)$ is 6 , personalization, and objectivization $(\mathrm{H} 6)$ is 1 , and facility of expression $(\mathrm{H} 7)$ is 24 .

Tabel 2

Analysis of the use of code-switching and code-mixing "How To Tips Memilih Sepatu Sesuai Bentuk Kaki" episode

\begin{tabular}{|c|c|c|c|c|}
\hline No & The Data & Lexical Form & Form & Function \\
\hline \multirow[t]{3}{*}{1.} & \multirow{3}{*}{$\begin{array}{l}\text { Sepatu merupakan fashion item yang di } \\
\text { sukai semua wanita, sampai sampai ada } \\
\text { quotes yang berbunyi "give a girl the right } \\
\text { shoes and she can conques the } \\
\text { world".Memilih sepatu sebenernya } \\
\text { gampang gampang susah, selain dengan } \\
\text { warna dan modelnya harus pas betuk } \\
\text { kaki juga sangat mempengaruhi. }\end{array}$} & Fashion item & G2 & $\mathrm{H} 7$ \\
\hline & & Quotes & G1 & $\mathrm{H} 7$ \\
\hline & & $\begin{array}{l}\text { "give a girl the } \\
\text { right shoes and } \\
\text { she can conques } \\
\text { the world". }\end{array}$ & F3 & $\mathrm{H} 1$ \\
\hline \multirow[t]{3}{*}{2.} & \multirow{3}{*}{$\begin{array}{l}\text { Berikut tips memilih sepatu sesuai } \\
\text { dengan bentuk kaki. Let's check this out! } \\
\text { I LOOK punya beberapa tips untuk memilih } \\
\text { sepatu sesuai dengan bentuk kaki. First, } \\
\text { bagi wanita yang memiliki pergelangan } \\
\text { kaki tebal ada baiknya menghindari } \\
\text { penggunaan wedges yang mengganggu, } \\
\text { akan lebih baik kamu memilih sandal } \\
\text { yang memiliki heel lancip. }\end{array}$} & $\begin{array}{l}\text { Let's check this } \\
\text { out! }\end{array}$ & F1 & $\mathrm{H} 4$ \\
\hline & & First & G1 & $\mathrm{H} 7$ \\
\hline & & Heel & G1 & $\mathrm{H} 7$ \\
\hline 3. & $\begin{array}{l}\text { Next, banyak wanita yang kurang percaya } \\
\text { diri karena memikliki betis yang tebal, } \\
\text { mereka pun merasa bahwa hampir } \\
\text { semua sepatu tidak cocok dengan }\end{array}$ & Next & G1 & $\mathrm{H} 7$ \\
\hline
\end{tabular}




\begin{tabular}{|c|c|c|c|c|}
\hline & $\begin{array}{l}\text { bentuk kaki mereka. } \\
\text { Well, yang harus kamu lakukan } \\
\text { menghindari penggunaan sepatu dengan } \\
\text { tali di perelangan kaki atau sepatu } \\
\text { gladiator. }\end{array}$ & Well & G1 & $\mathrm{H} 7$ \\
\hline \multirow[t]{2}{*}{4.} & \multirow{2}{*}{$\begin{array}{l}\text { Beberapa sepatu yang bisa kamu gunakan } \\
\text { adalah sepatu model heels, sneakers, } \\
\text { atau sepatu boots lutut yang akan } \\
\text { menutupi betis. Next untuk kamu memiliki } \\
\text { pergelangan kaki yang kurus sebaiknya } \\
\text { kamu meghindari pemakaian wedges. }\end{array}$} & Heels & G1 & $\mathrm{H} 7$ \\
\hline & & Next & G1 & $\mathrm{H} 7$ \\
\hline \multirow[t]{3}{*}{5.} & \multirow{3}{*}{$\begin{array}{l}\text { Pilihan yang paling pas adalah sepatu flat, } \\
\text { ini akan membuat permukaan kaki terlihat } \\
\text { lebih luas dan tidak terlalu mencolok. And } \\
\text { the last gonna list, bagi kamu yang } \\
\text { mempunyai kaki panjang bisa banget } \\
\text { mecoba menggunakan sepatu datar } \\
\text { seperti dengan tali di pergelangan kaki } \\
\text { seperti sendal gladiator dan engkel boots. } \\
\text { Hindari menggunakan heels karena akan } \\
\text { membuat kaki kamu semakin tinggi. Well, } \\
\text { itu dia tadi beberapa tips untuk memilih } \\
\text { sepatu sesuai dengan bentuk kaki }\end{array}$} & Flat & G1 & $\mathrm{H} 7$ \\
\hline & & $\begin{array}{c}\text { And the last } \\
\text { gonna list }\end{array}$ & F3 & $\mathrm{H} 5$ \\
\hline & & Well & G1 & $\mathrm{H} 7$ \\
\hline
\end{tabular}

From the table above, it can be seen that there are 5 occurrences of code-switching, the writer only found two types of code-switching, they are tag switching (F1) is 1, and intra-sential (F3) is 2. Meanwhile, there are 11 occurrences of code-mixing, the writer just found two types of code-mixing. They are the insertion of the word (G1) is 9, and the insertion of the phrase (G2) is 2. Then for the function of code-switching and code-mixing, the writer only found four functions in the "How to tips memilih sepatu sesuai bentuk kaki" episode. They are quotation $(H 1)$ is 1 , interjection $(H 4)$ is 1 , message qualification (H5) is 1 , and facility of expression $(\mathrm{H} 7)$ is 10 .

\section{Tabel 3}

Analysis of the use of code-switching and code-mixing "Tips Makeup Tahan Lama Untuk Kulit Berminyak" episode

\begin{tabular}{|c|c|c|c|c|}
\hline No & The Data & Lexical Form & Form & Function \\
\hline 1. & $\begin{array}{l}\text { Wahhh senengnya Kimcy mau nyobain } \\
\text { makeup baru ah. Hah! Aduh ya ampun } \\
\text { kok makeup Kimcy bolong bolong kaya } \\
\text { gini sih? Apa karena kulit Kimcy } \\
\text { berminyak ya? Jadi makeup Kimcy } \\
\text { cepet luntur. Hampir semua wanita } \\
\text { menggunakan makeup untuk tampil } \\
\text { cantik, tapi untu wajah yang berminyak } \\
\text { makeup cepat luntur, alhasil makeup } \\
\text { kita jadi sia sia. }\end{array}$ & Makeup & G1 & $\mathrm{H} 7$ \\
\hline \multirow[t]{3}{*}{2.} & \multirow{3}{*}{$\begin{array}{l}\text { Berikut ada beberapa tips yang dapat } \\
\text { kamu lakukan agar makeup kamu tahan } \\
\text { lama. Let's check this out! Banyak wanita } \\
\text { yang menggunakan makeup untuk tampil } \\
\text { cantik, tapi untuk wajah yang berminyak } \\
\text { kadang makeup cepat luntur, alhasil } \\
\text { makeup kita jadi sia-sia. I LOOK punya } \\
\text { tips untuk kulit wajah yang berminyak. At } \\
\text { the first makeup Primer. }\end{array}$} & $\begin{array}{c}\text { Let's check this } \\
\text { out! }\end{array}$ & $\mathrm{F} 1$ & $\mathrm{H} 4$ \\
\hline & & Makeup & G1 & $\mathrm{H} 7$ \\
\hline & & $\begin{array}{c}\text { At the first } \\
\text { makeup Primer }\end{array}$ & $\mathrm{F} 2$ & $\mathrm{H} 5$ \\
\hline \multirow[t]{3}{*}{3.} & \multirow{3}{*}{$\begin{array}{l}\text { Sebelum mengaplikasikan makeup } \\
\text { gunakan primer terlebih dahulu di area T } \\
\text { wajah atau } T \text { zone. T zone merupakan } \\
\text { bagian yang paling sering berminyak } \\
\text { yaitu bagian hidung, dahi, dan dagu. } \\
\text { Gunakan primer untuk khusus wajah } \\
\text { berminyak. Secound facial wash for oil }\end{array}$} & Makeup & G1 & $\mathrm{H7}$ \\
\hline & & $T$ zone & G2 & $\mathrm{H} 7$ \\
\hline & & Secound facial & F2 & $\mathrm{H} 5$ \\
\hline
\end{tabular}




\begin{tabular}{|c|c|c|c|c|}
\hline & skin. & wash for oil skin & & \\
\hline \multirow[t]{3}{*}{4.} & \multirow{3}{*}{$\begin{array}{l}\text { Saat ini sudah banyak branch kosmetik } \\
\text { yang membuat facial wash khusus kulit } \\
\text { berminyak, selain aman produk ini juga } \\
\text { biasanya lebih tahan lama untuk kulit } \\
\text { yang berminyak yang cenderung } \\
\text { lembab. Cara membedakan produk } \\
\text { khusus kulit berminyak dengan melihat } \\
\text { lebel oil free di kemasannya }\end{array}$} & Branch & G1 & $\mathrm{H} 7$ \\
\hline & & Facial wash & G2 & $\mathrm{H} 7$ \\
\hline & & Oil free & G2 & $\mathrm{H} 7$ \\
\hline \multirow[t]{5}{*}{5.} & \multirow{5}{*}{$\begin{array}{l}\text { And the last gonna list, blotting paper. } \\
\text { Ketika wajah kamu mulai berminyak dan } \\
\text { terlihat mengkilap jangan buru buru } \\
\text { menambah lapisan bedak pada wajah. } \\
\text { Bedak yang di aplikasikan pada wajah } \\
\text { saat berminyak bisa menyebabkan } \\
\text { olesan bedak tidak merata, lebih baik } \\
\text { gunakan blotting paper atau kertas yang } \\
\text { khusus untuk menyapu minyak pada } \\
\text { wajah. So ga usah khawatir fashion } \\
\text { people untuk kamu yang memiliki tipe } \\
\text { wajah berminyak, dengan tiga hal } \\
\text { simple tadi kamu bisa tampil lebih lama. } \\
\text { Happy trying! }\end{array}$} & $\begin{array}{c}\text { And the last } \\
\text { gonna list, blotting } \\
\text { paper }\end{array}$ & $\mathrm{F} 2$ & H5 \\
\hline & & Blotting paper & G2 & $\mathrm{H} 7$ \\
\hline & & So & G1 & $\mathrm{H} 7$ \\
\hline & & Fashion people & G2 & $\mathrm{H} 2$ \\
\hline & & Happy trying! & $\mathrm{F} 1$ & $\mathrm{H} 4$ \\
\hline
\end{tabular}

From the table above, it can be seen that there are 5 occurrences of code-switching, the writer only found two types of code-switching, they are tag switching (F1) is 2, and intra-sential (F2) is 3 . Meanwhile, there are 10 occurrences of code-mixing, the writer just found two types of code-mixing. They are the insertion of the word (G1) is 5 , and the insertion of the phrase (G2) is 5 . Then for the function of code-switching and code-mixing, the writer only found four functions in the "Tips makeup tahan lama untuk kulit berminyak" episode. They are addressee specification (H2) is 1, interjection $(\mathrm{H} 4)$ is 2 , message qualification (H5) is 3 , and facility of expression $(\mathrm{H} 7)$ is 9 .

Tabel 4

Analysis of the use of code-switching and code-mixing "Jenis Celana Yang Cocok Untuk Inner Skirt" episode

\begin{tabular}{|c|c|c|c|c|}
\hline No & The Data & Lexical Form & Form & Function \\
\hline 1. & $\begin{array}{l}\text { Ah ga sabar deh ngasih bingkisan ini } \\
\text { buat temen Kimcy, soalnya dia } \\
\text { kemarin ngajakin Kimcy beli inner skirt } \\
\text { tapi Kimcy ga bisa. Semoga inner skirt } \\
\text { pas buat dia. }\end{array}$ & Inner skirt & G2 & $\mathrm{H} 7$ \\
\hline \multirow[t]{4}{*}{2.} & \multirow{4}{*}{$\begin{array}{l}\text { Rok adalah salah satu fashion item } \\
\text { yang tidak boleh di lewatkan oleh } \\
\text { para hijaber, karena tidak hanya } \\
\text { sebagai luaran, rok juga di gunakan } \\
\text { sebagai inner dalam berbusana. } \\
\text { Masalahnya ketika menggunakn rok } \\
\text { para hijabers sering sekali kesulitan } \\
\text { untuk bergerak, untuk mengatasi hal } \\
\text { itu biasanya fashion people harus } \\
\text { menggunakan inner skirt ataupun } \\
\text { yang biasanya disebut dengan } \\
\text { dalaman }\end{array}$} & Fashion item & G2 & $\mathrm{H} 7$ \\
\hline & & Inner & G1 & $\mathrm{H} 7$ \\
\hline & & Fashion people & G2 & $\mathrm{H} 2$ \\
\hline & & Inner skirt & G2 & $\mathrm{H} 7$ \\
\hline \multirow[t]{3}{*}{3.} & \multirow{3}{*}{$\begin{array}{l}\text { Menggunakan inner skirt ataupun } \\
\text { yang biasanya disebut dengan } \\
\text { dalaman, but for your information } \\
\text { tidak semua cocok untuk inner skirt. } \\
\text { Berikut adalah jenis-jenis celana yang } \\
\text { mudah di gunakan untuk inner skrit. } \\
\text { Let's check this out! }\end{array}$} & $\begin{array}{l}\text { But for your } \\
\text { information }\end{array}$ & F3 & $\mathrm{H} 5$ \\
\hline & & Inner skirt & G2 & H3 \\
\hline & & Let's check this out! & $\mathrm{F} 1$ & $\mathrm{H} 4$ \\
\hline
\end{tabular}




\begin{tabular}{|c|c|c|c|c|}
\hline \multirow[t]{4}{*}{4.} & \multirow{4}{*}{$\begin{array}{l}\text { First leging. Leging adalah jenis celana } \\
\text { yang paling populer yang biasa di } \\
\text { gunakan untuk para hijabers. Pilihlah } \\
\text { celana yang full cotton spandex agar } \\
\text { kaki kalian tidak berkeringat saat } \\
\text { bergerak. Celana yang elastis ini } \\
\text { cocock di padu padankan untuk rok } \\
\text { apa saja bahkan gamis sekalipun, put } \\
\text { wear apapun juga gampang dengan } \\
\text { inner skirt yang satu ini }\end{array}$} & First & G1 & $\mathrm{H} 7$ \\
\hline & & full cotton spandex & F3 & $\mathrm{H} 7$ \\
\hline & & Put wear & F3 & $\mathrm{H} 7$ \\
\hline & & Inner skirt & G2 & $\mathrm{H} 7$ \\
\hline \multirow[t]{6}{*}{5.} & \multirow{6}{*}{$\begin{array}{l}\text { Next celana katun. Celana katun } \\
\text { memang tidak seelastis leging untuk } \\
\text { inner skrit. Celana katun cocok sekali } \\
\text { di mix and match dengan rok ataupun } \\
\text { rok berbahan katun juga, untuk kesan } \\
\text { yang lebih formal kalian juga bisa } \\
\text { menggunakan flat shoes. Next jeans. } \\
\text { Celana ini sangat sangat netral bisa } \\
\text { dipakai kapan saja dan dimana saja. } \\
\text { Apalagi untuk fashion people yang } \\
\text { memiliki kegiatan luar biasa sibuk }\end{array}$} & Next & G1 & $\mathrm{H} 7$ \\
\hline & & Inner skrit & G2 & $\mathrm{H} 7$ \\
\hline & & Mix and match & F3 & $\mathrm{H} 7$ \\
\hline & & Flat shoes & G2 & $\mathrm{H} 7$ \\
\hline & & Next & G1 & $\mathrm{H} 7$ \\
\hline & & Fashion people & G2 & $\mathrm{H} 2$ \\
\hline \multirow[t]{3}{*}{6.} & \multirow{3}{*}{$\begin{array}{l}\text { Untuk di negara tropis seperti } \\
\text { Indonesia dalaman jeans sangat } \\
\text { cocok untuk dalaman rok atau dress } \\
\text { tipis. Next kulot, bila kalian memiliki } \\
\text { rok ataupun dress yang sedikit blus } \\
\text { maupun mengembang, kulot adalah } \\
\text { merupakan inner skirt yang paling } \\
\text { tepat untuk kalian pilih, yang perlu di } \\
\text { perhatikan adalah dikarenakan dress } \\
\text { dan kulot sudah memberikan kesan } \\
\text { yang mengembangkan, maka kamu } \\
\text { harus memilih jilbab yang } \\
\text { sedarhana, baik dari motif, warna } \\
\text {,bahan ataupun cara pemakaiannya. }\end{array}$} & Dress & G1 & $\mathrm{H} 7$ \\
\hline & & Next & G1 & $\mathrm{H} 7$ \\
\hline & & Inner skrit & G2 & $\mathrm{H} 7$ \\
\hline \multirow[t]{5}{*}{7.} & \multirow{5}{*}{$\begin{array}{l}\text { Hal ini untuk menghindari penampilan } \\
\text { kalian yang terlalua penuh ataupun } \\
\text { menumpuk. And the last jogger pants. } \\
\text { Buat kalian yang ingin kelihatan } \\
\text { sporty, jogger pants adalah pilihan } \\
\text { yang tepat, tetapi yang perlu di ingat } \\
\text { kalian harus menyesuaikan rok } \\
\text { ataupun baju kalian dengan sepatu } \\
\text { yang ingin di pakai. Nah itu dia lima } \\
\text { jenis celana yang bisa kalian jadikan } \\
\text { inner skirt untuk para hijabers. Happy } \\
\text { weekand fashion people. }\end{array}$} & $\begin{array}{c}\text { And the last jogger } \\
\text { pants }\end{array}$ & $\mathrm{F} 1$ & $\mathrm{H} 7$ \\
\hline & & Sporty & G1 & $\mathrm{H} 7$ \\
\hline & & Jogger pants & G2 & $\mathrm{H} 7$ \\
\hline & & Inner skirt & G2 & $\mathrm{H} 7$ \\
\hline & & $\begin{array}{l}\text { Happy weekand } \\
\text { fashion people! }\end{array}$ & $\mathrm{F} 1$ & $\mathrm{H} 4$ \\
\hline
\end{tabular}

From the table above, it can be seen that there are 6 occurrences of code-switching, the writer only found two types of code-switching, they are tag switching (F1) is 2, and intra-sential (F3) is 4. Meanwhile, there are 19 occurrences of code-mixing, the writer just found two types of code-mixing. They are the insertion of the word (G1) is 7, and the insertion of the phrase (G2) is 12. Then for the function of code-switching and code-mixing the writer only found five function in "Jenis celana yang cocok untuk inner skirt" episode. They are addressee specification $(\mathrm{H} 2)$ is 2, repetition $(\mathrm{H} 3)$ is 1 , interjection ( $(\mathrm{H} 4)$ is 2, message qualification (H5) is 1, and facility of expression (H7) is 20.

\section{Tabel 5}

Analysis of code switching and code mixing "Walk In Closet Kimmy Jayanti" episode

\begin{tabular}{|l|l|l|l|l} 
No & The Data & Lexical Form & Form & Function \\
\hline
\end{tabular}




\begin{tabular}{|c|c|c|c|c|}
\hline \multirow[t]{5}{*}{1.} & \multirow{5}{*}{$\begin{array}{l}\text { Hai fashion people! Selamat datang } \\
\text { dirumah saya. Pasti penasaran } \\
\text { dengan item-item favorit saya. } \\
\text { Wanna see my walk in closet? } \\
\text { Come with me. Penasarankan } \\
\text { fashion people dirumah, } \\
\text { selanjutnya kita akan melihat the } \\
\text { secret of Kimmy Jayanti. Come with } \\
\text { me. }\end{array}$} & Hai fashion people! & $\mathrm{F} 1$ & $\mathrm{H} 4$ \\
\hline & & item-item & G4 & $\mathrm{H} 7$ \\
\hline & & $\begin{array}{c}\text { Wanna see my walk in } \\
\text { closet? }\end{array}$ & $\mathrm{F} 1$ & $\mathrm{H} 4$ \\
\hline & & Come with me & $\mathrm{F} 1$ & $\mathrm{H} 4$ \\
\hline & & $\begin{array}{c}\text { the secret of Kimmy } \\
\text { Jayanti. }\end{array}$ & F3 & $\mathrm{H} 2$ \\
\hline \multirow[t]{2}{*}{2.} & \multirow{2}{*}{$\begin{array}{l}\text { Disini saya ada beberapa koleksi } \\
\text { sepatu saya, mungkin bisa dilihat } \\
\text { disini ada beberapa, yang paling } \\
\text { favorit saya yang paling saya pakai } \\
\text { yang sering banget itu adalah ini, I } \\
\text { love it so much, selain maksudnya } \\
\text { membuat kaki saya keliahatan sexy } \\
\text { terus aku juga suka banget dengan } \\
\text { warnanya dan dapetinya juga } \\
\text { susah banget. }\end{array}$} & I love it so much & F3 & $\mathrm{H} 6$ \\
\hline & & Sexy & G1 & $\mathrm{H} 7$ \\
\hline \multirow[t]{3}{*}{3.} & \multirow{3}{*}{$\begin{array}{l}\text { Terus ada yang paling uniqe nih } \\
\text { para fashion people dirumah nih } \\
\text { lihat nih fashion people ini sepatu } \\
\text { buat sky, sampai sekarang saya } \\
\text { suka banget sama sepatu ini dan } \\
\text { ini juga menjadi favorit saya juga. } \\
\text { Koleksi saya juga untuk dipakai } \\
\text { sehari-hari, ga banayk sih fashion } \\
\text { people cuma yang paling aku suka } \\
\text { sih yang ini karena dapetinnya juga } \\
\text { susah }\end{array}$} & Uniqe & G1 & $\mathrm{H} 7$ \\
\hline & & fashion people & G2 & $\mathrm{H} 2$ \\
\hline & & Sky & G1 & $\mathrm{H} 7$ \\
\hline \multirow[t]{7}{*}{4.} & \multirow{7}{*}{$\begin{array}{l}\text { Terus aku juga biasanya pakai ke } \\
\text { red carpet red carpet gitu, yang ini } \\
\text { aku juga suka banget transparant } \\
\text { gitu, keren banget ya fashion } \\
\text { people jadi aku biasanya kalo mau } \\
\text { masukin makeup juga segala } \\
\text { sesuatu item item yang lebih } \\
\text { fancy aku masukin disini. Terus } \\
\text { terakhir ini fashion people, ini juga } \\
\text { yang paling saya suka kalo } \\
\text { misalnya saya mau traveling } \\
\text { karena semuanya bisa masuk ke } \\
\text { dalam sini, Kely Hermes lucu ya } \\
\text { fashion people }\end{array}$} & red carpet red carpet & G4 & $\mathrm{H} 7$ \\
\hline & & Transparant & G1 & $\mathrm{H} 7$ \\
\hline & & Makeup & G1 & $\mathrm{H} 7$ \\
\hline & & item item & G4 & $\mathrm{H} 7$ \\
\hline & & fancy & G1 & $\mathrm{H} 7$ \\
\hline & & fashion people & G2 & $\mathrm{H} 2$ \\
\hline & & traveling & G1 & $\mathrm{H} 7$ \\
\hline \multirow[t]{3}{*}{5.} & \multirow{3}{*}{$\begin{array}{l}\text { Kely Hermes lucu ya fashion } \\
\text { people. Kacamata saya disini ada } \\
\text { banyak banget ya, aku suka yang } \\
\text { ini, banyak kalo kacamata mah } \\
\text { that all my favorite. Kalo koleksi } \\
\text { kacamata sih fashion people saya }\end{array}$} & fashion people & G2 & $\mathrm{H} 2$ \\
\hline & & that all my favorite & F3 & $\mathrm{H} 6$ \\
\hline & & Sky & G1 & $\mathrm{H} 7$ \\
\hline
\end{tabular}


Renita Wati

\begin{tabular}{|c|c|c|c|c|}
\hline & $\begin{array}{l}\text { demen banget dengan kacamata, } \\
\text { lihat deh ini ada kacamata sky, ga } \\
\text { deng ini Tomfor fashion people. }\end{array}$ & Look & G1 & $\mathrm{H} 7$ \\
\hline & $\begin{array}{l}\text { Ada yang dilapisi emas ini lidah } \\
\text { fero. Saya memang demen demen } \\
\text { banget pake kacamata fashion } \\
\text { people untuk membuat look saya } \\
\text { jauh lebih styles sehari-hari }\end{array}$ & Styles & G1 & $\mathrm{H} 7$ \\
\hline \multirow[t]{7}{*}{6.} & \multirow{7}{*}{$\begin{array}{l}\text { Nah fashion people kalo tadi baju } \\
\text { baju casual kalo ini lebih ke winter } \\
\text { and evening goun. Evening goun } \\
\text { yang paling saya suka adalah ini, } \\
\text { koleksi dari Zahura Murad fashion } \\
\text { people, sampai sekarang saya ga } \\
\text { pernah pakai karena saya ga tau } \\
\text { mau di pakai kemana, keren } \\
\text { banget ya fashion people. I love all } \\
\text { the detail. I love it so much dan } \\
\text { bagus banget kalo misalnaya } \\
\text { dipakai untuk evening goun }\end{array}$} & fashion people & G2 & $\mathrm{H} 2$ \\
\hline & & Casual & G1 & $\mathrm{H} 7$ \\
\hline & & winter and evening goun & F3 & $\mathrm{H} 7$ \\
\hline & & Evening goun & G2 & $\mathrm{H} 7$ \\
\hline & & fashion people & G2 & $\mathrm{H} 2$ \\
\hline & & I love all the detail & $\mathrm{F} 2$ & $\mathrm{H} 6$ \\
\hline & & I love it so much & F3 & $\mathrm{H} 6$ \\
\hline \multirow[t]{7}{*}{7.} & \multirow{7}{*}{$\begin{array}{l}\text { Terus kalo baju baju winternya } \\
\text { juga aku banyak juga, buat ini } \\
\text { karena saya demen banget sama } \\
\text { treveling fashion people. Another } \\
\text { evering goun, I love it too, nih keren } \\
\text { keren banget kan, ini saya pakai } \\
\text { kalo ada red karpet gitu. Jewelry } \\
\text { favorit saya fashion peopel. Disini } \\
\text { ada cincin dan juga my favorite is } \\
\text { this one, coker diamon }\end{array}$} & Winternya & G3 & $\mathrm{H} 7$ \\
\hline & & treveling fashion people & F3 & $\mathrm{H} 7$ \\
\hline & & Another evering goun, & F3 & $\mathrm{H} 7$ \\
\hline & & I love it too & F3 & $\mathrm{H} 6$ \\
\hline & & Red & G1 & $\mathrm{H} 7$ \\
\hline & & fashion peopel & G2 & $\mathrm{H} 2$ \\
\hline & & $\begin{array}{c}\text { my favorite is this one } \\
\text { coker diamon }\end{array}$ & F3 & $\mathrm{H} 6$ \\
\hline \multirow[t]{3}{*}{8.} & \multirow{3}{*}{$\begin{array}{l}\text { Terus kalo buat sehari hari nih } \\
\text { fashion people lebih ke warna } \\
\text { warna gold gitu ya kan? Taraaa ini } \\
\text { juga tempat favorit aku fashion } \\
\text { people, karena disini adalah } \\
\text { tempat wewangian alias Perfume. }\end{array}$} & fashion people & G2 & $\mathrm{H} 2$ \\
\hline & & Gold & G1 & $\mathrm{H} 7$ \\
\hline & & Perfume & G1 & $\mathrm{H} 7$ \\
\hline \multirow[t]{8}{*}{9.} & \multirow{8}{*}{$\begin{array}{l}\text { Kenapa banyak banget karena } \\
\text { aku emang suka banget pake } \\
\text { parfum, jadi everyday itu aku } \\
\text { punya mood, kalo misalnya aku } \\
\text { sebelum berangkat kerja, kalo } \\
\text { misalnya hari ini aku merasa l'm } \\
\text { feeling glamor, so aku akan } \\
\text { menggunakan yang ini, parfum } \\
\text { yang ini Robbertocabali, terus kalo } \\
\text { misalnya the next day aku merasa } \\
\text { as a diva aku akan menggunakan } \\
\text { Cannel number five.l'm feeling } \\
\text { prety, I'm gonna use this as my } \\
\text { parfume Annaso. lihat nih dari } \\
\text { bentuknya aja udah lucu banget. }\end{array}$} & Everyday & G1 & $\mathrm{H} 7$ \\
\hline & & Mood & G1 & $\mathrm{H} 7$ \\
\hline & & I'm feeling glamor & F3 & $\mathrm{H} 6$ \\
\hline & & So & G1 & $\mathrm{H} 7$ \\
\hline & & the next day & G2 & $\mathrm{H} 7$ \\
\hline & & as a diva & G2 & $\mathrm{H} 7$ \\
\hline & & Cannel number five & F3 & $\mathrm{H} 2$ \\
\hline & & $\begin{array}{l}\text { I'm feeling prety, I'm } \\
\text { gonna use this as my } \\
\text { parfume Annaso, }\end{array}$ & $\mathrm{F} 2$ & $\mathrm{H} 6$ \\
\hline 10. & $\begin{array}{l}\text { Semoga bisa bermanfaat buat para } \\
\text { fashion people dirumah dan juga } \\
\text { jadi ga penasaran lagi kan? }\end{array}$ & Fashion People & G2 & $\mathrm{H} 2$ \\
\hline
\end{tabular}

From the table above, it can be seen that there are 14 occurrences of code-switching, the type in which tag switching (F1) is 3, inter-sential (F2) is 2, and intra-sential (F3) is 9. Meanwhile, there are 29 
occurrences of code-mixing, the writer just found four types of code-mixing. They are the insertion of the word (G1) is 15, the insertion of phrase (G2) is 10, the insertion hybrid (G3) is 1, and the insertion of reduplication (G4) is 3 . Then for the function of code-switching and code-mixing, the writer only found four functions in the " do and don't untuk kulit sawo matang " episode. They are addressee specification $(\mathrm{H} 2)$ is 9 , interjection $(\mathrm{H} 4)$ is 2 , personalization, and objectivization $(\mathrm{H} 6)$ is 8 , and facility of expression $(\mathrm{H} 7)$ is 27.

Tabel 6

Analysis of the use of code-switching and code-mixing "Tips Makeup Essential Sesuai Tingkatan Umur" episode

\begin{tabular}{|c|c|c|c|c|}
\hline No & $\begin{array}{c}\text { The Data } \\
\end{array}$ & Lexical Form & Form & Function \\
\hline 1. & $\begin{array}{l}\text { Kimcy mau ke mall nih nemeni } \\
\text { keponakan Kimcy yang sekarang udah } \\
\text { masuk kuliah dan pengen makeup. } \\
\text { Padahal dia itukan ga bisa makeup } \\
\text { lho dulu,sekarang udah tanya makeup. }\end{array}$ & Makeup & G1 & $\mathrm{H} 7$ \\
\hline \multirow[t]{3}{*}{2.} & \multirow{3}{*}{$\begin{array}{l}\text { Beberapa dari kita pasti sudah tidak } \\
\text { asing lagikan dengan yang namanya } \\
\text { makeup atau berbagai fashion item } \\
\text { yang lainnya fashion people, tapi harus } \\
\text { selalu diingat memilih makeup iru harus } \\
\text { sesuai dengan kebutuhan, karena selain } \\
\text { tampilan makeup kamu akan kelihatan } \\
\text { lebih keren }\end{array}$} & Makeup & G1 & $\mathrm{H} 7$ \\
\hline & & Fashion item & G2 & $\mathrm{H} 7$ \\
\hline & & Fashion people & G2 & $\mathrm{H} 2$ \\
\hline \multirow[t]{6}{*}{3.} & \multirow{6}{*}{$\begin{array}{l}\text { Nah I LOOK punya tipe makeup } \\
\text { essensial apa saja yang sesuai dengan } \\
\text { tingkatan umur kamu fashion people. } \\
\text { Let's check this out! First school girl. } \\
\text { Untuk kamu yang masih duduk } \\
\text { dibangku selolah makeup belum } \\
\text { diperlukan banget kok, karena kulit } \\
\text { kamu pada masa ini akan terlihat lebih } \\
\text { ok jika tanpa makeup ataupun riasan } \\
\text { yang natural }\end{array}$} & Fashion people & G2 & $\mathrm{H} 2$ \\
\hline & & Let's check this out! & F1 & $\mathrm{H} 4$ \\
\hline & & First school girl & F2 & $\mathrm{H} 2$ \\
\hline & & Makeup & G1 & $\mathrm{H} 7$ \\
\hline & & Ok & $\mathrm{Gi}$ & $\mathrm{H} 7$ \\
\hline & & Natural & G1 & $\mathrm{H} 7$ \\
\hline \multirow[t]{4}{*}{4.} & \multirow{4}{*}{$\begin{array}{ll}\text { Tetapi jika makeup sedang dibutuhkan } \\
\text { kamu bisa menggunakan makeup tipis } \\
\text { saja, blush on it's ok! } & \text { Untuk } \\
\text { menahan minyak diwajah dan } & \text { untuk } \\
\text { tampil lebih fresh di weekend } & \end{array}$} & Makeup & G1 & $\mathrm{H} 7$ \\
\hline & & Blush on it't ok! & $\mathrm{F} 1$ & $\mathrm{H} 4$ \\
\hline & & Fresh & G1 & $\mathrm{H} 7$ \\
\hline & & Weekend & G1 & $\mathrm{H} 5$ \\
\hline \multirow[t]{5}{*}{5.} & \multirow{5}{*}{$\begin{array}{l}\text { Kamu bisa menambahkan lip blam } \\
\text { ataupun lip tint dan juga blush on. Next } \\
\text { college girl. Ini dia tahapan diman } \\
\text { belajar makeup itu diperlukan ,karena } \\
\text { pada masa ini kalian lagi seru serunya } \\
\text { mencoba berbagai alat makeup, iya } \\
\text { kan? }\end{array}$} & Lip blam & G2 & $\mathrm{H} 7$ \\
\hline & & Lip tint & G2 & $\mathrm{H} 7$ \\
\hline & & Blush on & G2 & $\mathrm{H} 7$ \\
\hline & & Next college girl & $\mathrm{F} 2$ & $\mathrm{H} 2$ \\
\hline & & Makeup & G1 & $\mathrm{H} 7$ \\
\hline 6. & $\begin{array}{l}\text { Nah untuk tampilan sehari hari kamu } \\
\text { bisa mengandalkan tintimosterazer } \\
\text { dengan warna yang light untuk wajah }\end{array}$ & Light & G1 & $\mathrm{H} 7$ \\
\hline \multirow[t]{3}{*}{7.} & \multirow{3}{*}{$\begin{array}{l}\text { Jangan lupa tambahkan lipstick to } \\
\text { confirmed your look. And the last first } \\
\text { jobber. Tampilan makeup kamu } \\
\text { ditahap ini harus kace fashion people }\end{array}$} & $\begin{array}{c}\text { Lipstick to confirmed } \\
\text { your look }\end{array}$ & F3 & H5 \\
\hline & & $\begin{array}{c}\text { And the last first } \\
\text { jobber }\end{array}$ & F2 & $\mathrm{H} 2$ \\
\hline & & Fashion people & G2 & $\mathrm{H} 2$ \\
\hline \multirow[t]{2}{*}{8.} & \multirow{2}{*}{$\begin{array}{l}\text { Ini saatnya kamu bisa memakai } \\
\text { makeup yang base seperti primer, } \\
\text { foundation atau setting makeup yang } \\
\text { biasa membuat kamu terlihat flawless } \\
\text { seharian }\end{array}$} & Setting makeup & F3 & $\mathrm{H} 7$ \\
\hline & & Flawless & G1 & $\mathrm{H} 7$ \\
\hline
\end{tabular}




\begin{tabular}{|c|c|c|c|c|}
\hline \multirow[t]{4}{*}{9.} & \multirow{4}{*}{$\begin{array}{l}\text { Untuk riasan mata pilihlah nuansa yang } \\
\text { warm seperti coklat dan padukan } \\
\text { dengan bulu mata palsu yang tebal } \\
\text { agar mata kalian terlihat lebih hidup. Itu } \\
\text { dia beberapa tips makeup essensial } \\
\text { yang sesuai dengan tingkatan umur } \\
\text { kamu. Wanna more about tips? stay } \\
\text { tuned on I LOOK }\end{array}$} & Warm & G1 & $\mathrm{H} 7$ \\
\hline & & Makeup essensial & F3 & H5 \\
\hline & & $\begin{array}{c}\text { Wanna more about } \\
\text { tips? }\end{array}$ & F1 & $\mathrm{H} 4$ \\
\hline & & $\begin{array}{c}\text { Stay tuned on I } \\
\text { LOOK! }\end{array}$ & F1 & $\mathrm{H} 4$ \\
\hline
\end{tabular}

From the table above, it can be seen that there are 10 occurrences of code-switching, the type in which tag switching (F1) is 4 , inter-sential (F2) is 3 , and intra-sential (F3) is 3 . Meanwhile, there are 18 occurrences of code-mixing, the writer just found two types of code-mixing. They are the insertion of the word (G1) is 11, and the insertion of the phrase (G2) is 3. Then for the function of code-switching and code-mixing, the writer only found four function in "tips makeup essential sesuai tingkatan umur" episode. They are addressee specification ( $\mathrm{H} 2)$ is 3 , interjection $(\mathrm{H} 4)$ is 4, message qualification $(\mathrm{H} 5)$ is 3 , and facility of expression (H7) is 15 .

Tabel 7

Analysis of code-switching and code mixing in "Do And Don't Untuk Kulit Sawo Matang" episode

\begin{tabular}{|c|c|c|c|c|}
\hline No & The Data & Lexical Form & Form & Function \\
\hline 1. & $\begin{array}{l}\text { Cewek dengan warna alami kulit sawo } \\
\text { matang di Indonesia sudah hal biasa, } \\
\text { tidak seperti orang orang di negara } \\
\text { barat kulit sawo matang ini bisa di } \\
\text { dapatkan tanpa harus tan, tapi } \\
\text { terkadang beberapa dari kamu suka } \\
\text { ga Pede dengan kulit sawo matang }\end{array}$ & Tan & G1 & $\mathrm{H} 7$ \\
\hline \multirow[t]{6}{*}{2.} & \multirow{6}{*}{$\begin{array}{l}\text { Jenis kulit kamu sawo matang fashion } \\
\text { people? Don't worry! Kamu ga perlu } \\
\text { minder sama kulit kita karena anggapan } \\
\text { cantik itu harus punya warna kulit } \\
\text { terang, it's a big mistake girl! We must } \\
\text { pround of it, karena kulit sawo matang } \\
\text { adalah warna kulit alami bagi } \\
\text { masyarakat Indonesia dan lebih sering } \\
\text { disebut sebagai olive skintone }\end{array}$} & Fashion peoe & G2 & $\mathrm{H} 2$ \\
\hline & & Don't worry! & $\mathrm{F} 1$ & $\mathrm{H} 4$ \\
\hline & & Tanning & G1 & $\mathrm{H} 7$ \\
\hline & & $\begin{array}{c}\text { It's a big mistake } \\
\text { girl! }\end{array}$ & $\mathrm{F} 1$ & $\mathrm{H} 4$ \\
\hline & & $\begin{array}{c}\text { We must pround of } \\
\text { it }\end{array}$ & F3 & $\mathrm{H} 6$ \\
\hline & & Olive skintone & G2 & $\mathrm{H} 7$ \\
\hline \multirow[t]{2}{*}{3.} & \multirow{2}{*}{$\begin{array}{l}\text { Kamu bisa tonjolin kecantikannya } \\
\text { dengan do and don't yang satu ini. } \\
\text { Let's check this out! }\end{array}$} & Do and don't & F3 & $\mathrm{H} 7$ \\
\hline & & Let's check this out! & $\mathrm{F} 1$ & $\mathrm{H} 4$ \\
\hline \multirow[t]{3}{*}{4.} & \multirow{3}{*}{$\begin{array}{l}\text { Perfect foundation color, this thing a bit } \\
\text { littel tricky. Kamu hindari makeup base } \\
\text { seperti foundation atau BB cream yang } \\
\text { warnanya lebih terang dari warna kulit } \\
\text { kamu, base dengan warna yang tidak } \\
\text { sesuai akan membuat makeup kamu } \\
\text { kelihatan too much fashion people }\end{array}$} & $\begin{array}{l}\text { Perfect foundation } \\
\text { color, this thing a } \\
\text { bit littel tricky }\end{array}$ & $\mathrm{F} 2$ & $\mathrm{H} 6$ \\
\hline & & Makeup & G1 & $\mathrm{H} 7$ \\
\hline & & $\begin{array}{c}\text { Too much fashion } \\
\text { people }\end{array}$ & F3 & $\mathrm{H} 2$ \\
\hline \multirow[t]{2}{*}{5.} & \multirow{2}{*}{$\begin{array}{l}\text { Hightlight your face. Kulit sawo matang } \\
\text { yang berkilau bakal bikin tampilan kamu } \\
\text { makin kece, give glow to your skin } \\
\text { fashion people. }\end{array}$} & Hightlight your face & $\mathrm{F} 2$ & H5 \\
\hline & & $\begin{array}{c}\text { Give glow to your } \\
\text { skin fashion people. }\end{array}$ & F3 & $\mathrm{H} 6$ \\
\hline \multirow[t]{2}{*}{6.} & \multirow{2}{*}{$\begin{array}{l}\text { Using peachy coral colour. Peachy coral } \\
\text { for lips and cheek is one hunder persent } \\
\text { sweet your a lips skintone. Kamu bisa } \\
\text { pilih warna pink dengan warna soft soft } \\
\text { untuk kesan yang lebih fresh dan } \\
\text { natural. }\end{array}$} & $\begin{array}{c}\text { Using peachy coral } \\
\text { colour }\end{array}$ & $\mathrm{F} 2$ & H5 \\
\hline & & $\begin{array}{l}\text { Peachy coral for } \\
\text { lips and cheek is } \\
\text { one hunder persent } \\
\text { sweet your a lips } \\
\text { skintone }\end{array}$ & $\mathrm{F} 2$ & $\mathrm{H} 6$ \\
\hline
\end{tabular}




\begin{tabular}{|c|c|c|c|c|}
\hline & & Soft Soft & G4 & $\mathrm{H} 7$ \\
\hline & & Fresh & G1 & $\mathrm{H} 7$ \\
\hline & & Natural & G1 & $\mathrm{H} 7$ \\
\hline \multirow[t]{3}{*}{7.} & \multirow{3}{*}{$\begin{array}{l}\text { Bronzing your face. Kulit yang glowing } \\
\text { akan memberikan efek wajah yang lebih } \\
\text { terawat dan juga kece. Pasti kamu } \\
\text { sering melihat gimana kecenya hasil } \\
\text { bronzing beauty vloger. }\end{array}$} & Bronzing your face & $\mathrm{F} 2$ & $\mathrm{H} 5$ \\
\hline & & Glowing & G1 & $\mathrm{H} 7$ \\
\hline & & $\begin{array}{c}\text { Bronzing beauty } \\
\text { vloger }\end{array}$ & F3 & $\mathrm{H} 2$ \\
\hline \multirow[t]{5}{*}{8.} & \multirow{5}{*}{$\begin{array}{l}\text { Bronzing bisa membuat tampilan kulit } \\
\text { kamu menjadi makin gelap and it's not } \\
\text { feanturing added leadies. Choose pink } \\
\text { for eyeshadow and blush. It's ok to } \\
\text { wearing pink for olive skintone, tapi kalo } \\
\text { kamu ingin tampilan makeup jadi lebih } \\
\text { kelihatan kece baiknya kamu menjauhi } \\
\text { eyeshadow atau blash dengan warna } \\
\text { pink, }\end{array}$} & Bronzing & G1 & $\mathrm{H} 7$ \\
\hline & & $\begin{array}{l}\text { It's not feanturing } \\
\text { added leadies }\end{array}$ & F3 & $\mathrm{H} 6$ \\
\hline & & $\begin{array}{c}\text { Choose pink for } \\
\text { eyeshadow and } \\
\text { blush }\end{array}$ & $\mathrm{F} 2$ & $\mathrm{H} 5$ \\
\hline & & $\begin{array}{c}\text { It's ok to wearing } \\
\text { pink for olive } \\
\text { skintone }\end{array}$ & F3 & $\mathrm{H} 3$ \\
\hline & & Pink & G1 & $\mathrm{H} 7$ \\
\hline \multirow[t]{9}{*}{9.} & \multirow{9}{*}{$\begin{array}{l}\text { Ga hanya terlihat berlebihan di kulit } \\
\text { sawo matang kamu,warna pink juga } \\
\text { cenderung kelihatan ga menyatu } \\
\text { dengan undertone kulit kamu yang } \\
\text { sedikit sawo matang. Hesitate trying } \\
\text { deep color eyeshadow. Kalo kamu } \\
\text { suka natural makeup dengan warna } \\
\text { aman buat kulit sawo matang kamu } \\
\text { pastinya warna brown atau gold adalah } \\
\text { warna andalan kamu, trying something } \\
\text { different and buat mata kamu terlihat } \\
\text { brighten dengan warna yang lebih } \\
\text { berani. Nah itu dia do and don't buat } \\
\text { kamu yang memiliki kulit sawo matang. } \\
\text { Semoga info ini bermanfaat buat kamu } \\
\text { ya. Happy trying fashion people! }\end{array}$} & Pink & G1 & $\mathrm{H} 7$ \\
\hline & & Undertone & G1 & $\mathrm{H} 7$ \\
\hline & & $\begin{array}{c}\text { Hesitate trying } \\
\text { deep color } \\
\text { Eyeshadow }\end{array}$ & $\mathrm{F} 2$ & $\mathrm{H} 5$ \\
\hline & & Natural makeup & G2 & $\mathrm{H} 7$ \\
\hline & & Brown & G1 & $\mathrm{H} 7$ \\
\hline & & Gold & G1 & $\mathrm{H} 7$ \\
\hline & & $\begin{array}{l}\text { Trying something } \\
\text { different and }\end{array}$ & F3 & $\mathrm{H} 6$ \\
\hline & & Do and don't & F3 & $\mathrm{H} 7$ \\
\hline & & $\begin{array}{c}\text { Happy trying } \\
\text { fashion people! }\end{array}$ & $\mathrm{F} 1$ & $\mathrm{H} 4$ \\
\hline
\end{tabular}

From the table above, it can be seen that there are 20 occurrences of code-switching, the type which tags switching $(\mathrm{F} 1)$ is 4 , inter-sential $(\mathrm{F} 2)$ is 7 , and intra-sential (F3) is 9. Meanwhile, there are 15 occurrences of code-mixing, the writer just found three types of code-mixing. They are the insertion of the word (G1) is 11, the insertion of phrase (G2) is 3, and the insertion of reduplication (G4) is 1, and then for the function of code-switching and code-mixing, the writer only found five functions in " do and don't untuk kulit sawo matang " episode. They are addressee specification $(\mathrm{H} 2)$ is 3 , interjection $(\mathrm{H} 4)$ is 4 , message qualification $(\mathrm{H} 5)$ is 4 , personalization and objectivization $(\mathrm{H} 6)$ is 6 , and facility of expression $(\mathrm{H} 7)$ is 15 .

\section{CONCLUSION}

After analyzed the data as stated in the previous chapter of this study, the writer makes some conclusions based on the findings and the discussions as follows:

Based on the discussion in chapter IV, the writer can conclude that the total data 72 unit of analysis of code-switching found that 22 data used tag switching, 18 data used inter-sentential switching, and 32 used intra-sentential switching. It showed that intra-sentential switching is commonly used by Kimmy Jayanti in the fashion program "I LOOK" on NET TV. Furthermore, 127 unit data analysis of code-mixing performed 74 insertions of words, 48 insertions of phrases, 2 insertions of hybrids, and 3 insertions of reduplications. 
Meanwhile, Based on the data, there are 250 functions of code-switching and code-mixing used by Kimmy Jayanti in the fashin program "I LOOK" on NET TV. They are quotation $(\mathrm{H} 1)$ 1, addressee specification $(\mathrm{H} 2)$ 24, repetition $(\mathrm{H} 3)$ 1, interjection $(\mathrm{H} 4)$ 31, message qualification $(\mathrm{H} 5)$ 12, personalization, and objectivization (H6) 15, and facility of expression (H7) 121.

\section{REFERENCIES}

Crystal, D.(2003). Englis as a global language. $2^{\text {nd }}$ ed. Cambridge University Press

Jendra, M.I. (2012). SOCIOLINGUISTICS: Study of Societies" Languages. Yogyakarta: Graha IImu.

Kridalaksana, H. (2008). Kamus Linguistik. Jakarta: PT.Gramedia Pustaka Utama.

Marasigan, E. (1983). Code-Switching and Code-Mixing in multilingual societies: monograph series. Singapore: Singapore University Press.

Moleong, L. J., Prof., M.M. (2009). Metode Penelitian Kualitatif. Ed Revisi. Bandung: Remaja Rosdakarya.

Richards, J. (2002). Dictionary language Teaching\&Applied Linguistics: Longman.

Shite, D. R. (2016). Code mixing and code-switching used by English lectures at Jambi University. A Thesis Proposal. Jambi: Universitas Jambi.

Suwito. (1983). Pengantar Awal Sosiolinguistik: Teori dan Problema. Surakarta: Henary Offset Solo.

Wardhaugh, R. (2006). An Introduction to Sociolinguistics. $5^{\text {th }}$ ed. United Kingdom: Blackwell Publishing.

Wibowo, R. (2001). Language Management.New York: Scholastic. 